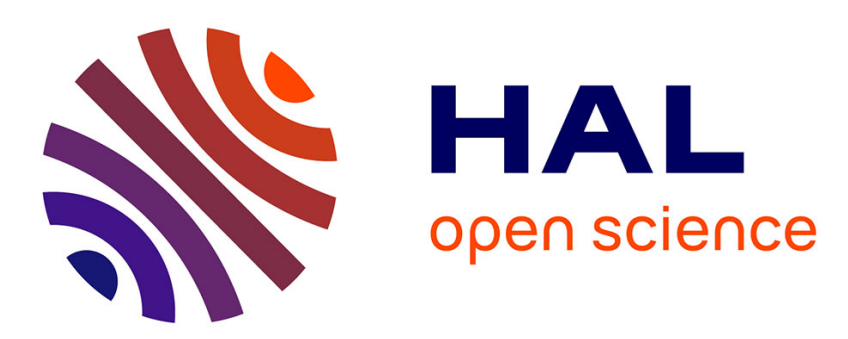

\title{
Two and three inputs Widely Linear FRESH receivers for cancellation of a quasi-rectilinear interference with frequency offset
}

\author{
Rémi Chauvat, Jean-Pierre Delmas, Pascal Chevalier
}

\section{To cite this version:}

Rémi Chauvat, Jean-Pierre Delmas, Pascal Chevalier. Two and three inputs Widely Linear FRESH receivers for cancellation of a quasi-rectilinear interference with frequency offset. EUSIPCO 2016: 24th European Signal Processing Conference, Aug 2016, Budapest, Hungary. pp.1398 - 1402, 10.1109/EUSIPCO.2016.7760478 . hal-01394314

\section{HAL Id: hal-01394314 \\ https://hal.science/hal-01394314}

Submitted on 9 Nov 2016

HAL is a multi-disciplinary open access archive for the deposit and dissemination of scientific research documents, whether they are published or not. The documents may come from teaching and research institutions in France or abroad, or from public or private research centers.
L'archive ouverte pluridisciplinaire HAL, est destinée au dépôt et à la diffusion de documents scientifiques de niveau recherche, publiés ou non, émanant des établissements d'enseignement et de recherche français ou étrangers, des laboratoires publics ou privés. 


\title{
Two and Three Inputs Widely Linear FRESH Receivers for Cancellation of a Quasi-Rectilinear Interference with Frequency Offset
}

\author{
Rémi Chauvat*†, Jean-Pierre Delmas ${ }^{\ddagger}$, Pascal Chevalier*† \\ ${ }^{*}$ CNAM, CEDRIC Laboratory, 292 rue Saint-Martin, 75141 Paris Cédex 3, France \\ ${ }^{\dagger}$ Thales-Communications-Security, HTE/AMS/TCP, 4 av. des Louvresses, 92622 Gennevilliers, France \\ ${ }^{\ddagger}$ Telecom SudParis, UMR CNRS 5157, 9 rue Charles Fourier, 91011 Evry, France
}

\begin{abstract}
Widely linear (WL) receivers can fulfill single antenna interference cancellation (SAIC) of one rectilinear (R) or quasi-rectilinear (QR) co-channel interference (CCI). The SAIC technology for $Q R$ signals has been shown to be less powerful than SAIC for $R$ signals. To overcome this limitation, a SAIC/MAIC enhancement using three-inputs WL frequency-shift (FRESH) receiver has been introduced for QR signals. However, this receiver loses its efficiency for an interference having a residual frequency offset (FO) above a fraction of the baud rate. This may appear for airborne communications and it is the case for the inter-carrier interference of filter-bank based multicarrier waveforms using OQAM constellations which are candidate for 5G mobile networks. This paper extends the standard two-inputs SAIC/MAIC receiver and the three-inputs WL FRESH receiver for QR signals with FO. Analytical results and simulations are presented to study the impact of this FO on the performance of these receivers.
\end{abstract}

\section{INTRODUCTION}

These two last decades, since the pioneer works on the subject [1-4], WL filtering has raised up a great interest for second-order (SO) non-circular signals [5] in many areas. Nevertheless, the subject which has received the greatest interest is CCI mitigation in radio communication networks using $\mathrm{R}$ or $\mathrm{QR}$ modulations. $\mathrm{R}$ modulations correspond to monodimensional modulations such as ASK or BPSK modulations. QR modulations are complex modulations corresponding, after a simple derotation operation, to a complex filtering of a $R$ modulation. Examples of QR modulations are MSK, GMSK or OQAM modulations. One of the most important properties of WL filtering is its ability to fulfill SAIC of one R or QR multi-user CCI, allowing the separation of two users from only one receive antenna [6-8]. The effectiveness of this technology jointly with its low complexity explain why it is operational in most of GSM handsets, allowing significant networks capacity gains for the GSM system [8]. Extension of the SAIC concept to a multi-antenna reception is called MAIC. However, it has been shown recently in [9] that the SAIC/MAIC technology for QR signals may be less powerful than the one for R signals. This result is directly related to the different cyclostationarity and non-circularity properties of $\mathrm{R}$ and $\mathrm{QR}$ signals. To overcome this limitation, a SAIC/MAIC enhancement based on the concept of three inputs WL FRESH receiver has been introduced in [10] for QR signals. However, it has been shown in [11] in the GSM context that SAIC/MAIC receivers, optimal in a minimum mean square error sense, lose their efficiency if, after the signal of interest (SOI) synchronization, the CCI has a residual FO above a small fraction of the baud rate. This may be the case for airborne communications, due to a potential high differential Doppler shift between the SOI and the CCI. This is also the case in the context of FBMC-OQAM waveforms, which are considered as promising candidates for 5G mobile networks in particular [12]. Indeed, the ICI of FBMC-OQAM waveforms, which is present at reception for highly frequency selective channels or for multiple-input multiple-output systems, have FO corresponding to multiple of $50 \%$ of the (real) baud rate. Note that the scarce WL filtering based solutions available for this problem [13], [14] or for CCI mitigation [15] are two-inputs WL receivers which do not exploit explicitly the FO information.

In this context, the purpose of this paper is twofold. The first one is to extend, for arbitrary propagation channels, both the standard two-inputs SAIC/MAIC receiver and the concept of three inputs WL FRESH receiver introduced in [10] for QR signals with non zero differential FO. The second one is to analyze, both analytically and by simulations, the impact of this differential FO on the performance of these two SAIC/MAIC receivers. Note that the scarce papers dealing with WL FRESH filtering for demodulation of QR signals [16-18] do not consider the proposed WL FRESH receivers and do not present any analysis of potential differential FO on the performance. To our knowledge, the impact of differential FO on the performance of WL receivers has been analyzed in [19] for R signals but has never been analysed for QR signals before this paper.

\section{Models AND SO STATistics}

\section{A. Observation model and SO statistics}

We consider an array of $N$ narrow-band antennas receiving the contribution of a QR SOI, one QR multi-user CCI and a background noise. The vector of complex amplitudes of the signals at the output of these antennas after frequency synchronization can then be written as

$$
\begin{aligned}
\mathbf{x}(t)=\sum_{k} j^{k} b_{k} \mathbf{g}(t-k T) & \\
& +\sum_{k} j^{k} e_{k}\left[v(t-k T) e^{j 2 \pi \Delta_{f} t}\right] * \mathbf{h}_{I}(t)+\mathbf{u}(t)
\end{aligned}
$$




$$
\begin{aligned}
& \mathbf{x}(t)=\sum_{k} j^{k} b_{k} \mathbf{g}(t-k T)+\sum_{k} j^{k} e_{k} e^{j 2 \pi \Delta_{f} k T} \mathbf{g}_{I_{o}}(t-k T)+\mathbf{u}(t) \\
& \mathbf{x}(t) \triangleq \sum_{k} j^{k} b_{k} \mathbf{g}(t-k T)+\mathbf{n}(t) .
\end{aligned}
$$

Here, $b_{k}$ and $e_{k}$ are real-valued zero-mean i.i.d. random variables, directly related to the SOI and CCI symbols respectively [7], [20], $T$ is the symbol period for MSK and GMSK signals and half the symbol period for OQAM signals, $\mathbf{g}(t)=v(t) * \mathbf{h}(t)$ is the impulse response of the SOI global channel, $*$ is the convolution operator, $v(t)$ and $\mathbf{h}(t)$ are the impulse responses of the SOI pulse shaping filter and propagation channel respectively, $\Delta_{f}$ is the residual FO of the CCI, which is assumed to be known (as for the ICI of FBMC-OQAM waveforms) or perfectly estimated a priori, $\mathbf{g}_{I_{o}}(t)=v_{o}(t) * \mathbf{h}_{I}(t)$ where $v_{o}(t)=v(t) e^{j 2 \pi \Delta_{f} t}$ and $\mathbf{h}_{I}(t)$ is the impulse response of the propagation channel of the $\mathrm{CCI}, \mathbf{u}(t)$ is the background noise vector, assumed zero-mean, circular, stationary, temporally and spatially white and $\mathbf{n}(t)$ is the total noise vector composed of the CCI and background noise. Note that model (1) is exact for MSK and OQAM signals whereas it is only an approximated model for GMSK signals.

The SO statistics of $\mathbf{n}(t)$ are characterized by the two correlation matrices $\mathbf{R}_{n}(t, \tau)$ and $\mathbf{C}_{n}(t, \tau)$, defined by

$$
\begin{aligned}
& \mathbf{R}_{n}(t, \tau) \triangleq \mathrm{E}\left[\mathbf{n}(t+\tau / 2) \mathbf{n}^{H}(t-\tau / 2)\right] \\
& \mathbf{C}_{n}(t, \tau) \triangleq \mathrm{E}\left[\mathbf{n}(t+\tau / 2) \mathbf{n}^{T}(t-\tau / 2)\right]
\end{aligned}
$$

where $(.)^{T}$ and $(.)^{H}$ mean transpose and conjugate transpose respectively. Using (1), it is easy to verify that $\mathbf{R}_{n}(t, \tau)$ is a periodic function of $t$ with period equal to $T$. In a same way, it is easy to show that $\mathbf{C}_{n}(t, \tau)=\mathbf{C}_{n}^{\prime}(t, \tau) e^{j 4 \pi \Delta_{f} t}$ where $\mathbf{C}_{n}^{\prime}(t, \tau)$ is a periodic function of $t$ with period equal to $2 T$. Matrices $\mathbf{R}_{n}(t, \tau)$ and $\mathbf{C}_{n}(t, \tau)$ have then Fourier series expansions given by

$$
\begin{aligned}
& \mathbf{R}_{n}(t, \tau)=\sum_{\alpha_{i}} \mathbf{R}_{n}^{\alpha_{i}}(\tau) e^{j 2 \pi \alpha_{i} t} \\
& \mathbf{C}_{n}(t, \tau)=\sum_{\beta_{i}} \mathbf{C}_{n}^{\beta_{i}}(\tau) e^{j 2 \pi \beta_{i} t} .
\end{aligned}
$$

Here, $\alpha_{i}$ and $\beta_{i}$ are the so-called non-conjugate and conjugate SO cyclic frequencies of $\mathbf{n}(t)$ such that $\alpha_{i}=i / T$ and $\beta_{i}=$ $(2 i+1) / 2 T+2 \Delta_{f}(i \in \mathbb{Z})[21], \mathbf{R}_{n}^{\alpha_{i}}(\tau)$ and $\mathbf{C}_{n}^{\beta_{i}}(\tau)$ are the first and second cyclic correlation matrices of $\mathbf{n}(t)$ for the cyclic frequencies $\alpha_{i}$ and $\beta_{i}$ and the delay $\tau$, defined by

$$
\begin{aligned}
& \mathbf{R}_{n}^{\alpha_{i}}(\tau) \triangleq\left\langle\mathbf{R}_{n}(t, \tau) e^{-j 2 \pi \alpha_{i} t}\right\rangle \\
& \mathbf{C}_{n}^{\beta_{i}}(\tau) \triangleq\left\langle\mathbf{C}_{n}(t, \tau) e^{-j 2 \pi \beta_{i} t}\right\rangle
\end{aligned}
$$

where $\langle\cdot\rangle$ is the temporal mean operation in $t$ over an infinite observation duration.

\section{B. Current two and three inputs FRESH model}

Conventional linear processing of $\mathbf{x}(t)$ [9], [10] only exploits the information contained in the zero non-conjugate $(\alpha=0)$ SO cyclic frequency of $\mathbf{x}(t)$.
Standard WL filtering of QR signals requires a derotation preprocessing to make $\mathrm{QR}$ signals look like $\mathrm{R}$ signals. Using (1), the derotated observation vector can be written as

$$
\mathbf{x}_{d}(t) \triangleq j^{-\frac{t}{T}} \mathbf{x}(t)=\sum_{k} b_{k} \mathbf{g}_{d}(t-k T)+\mathbf{n}_{d}(t)
$$

where $\mathbf{g}_{d}(t) \triangleq j^{-t / T} \mathbf{g}(t)$ and $\mathbf{n}_{d}(t) \triangleq j^{-t / T} \mathbf{n}(t)$. Standard WL filtering of $\mathbf{x}_{d}(t)$ only exploits the information contained in the zero non-conjugate and conjugate $\left(\alpha_{d}, \beta_{d}\right)=$ $(0,0)$ SO cyclic frequencies of $\mathbf{x}_{d}(t)$, or equivalently in the SO cyclic frequencies, $(\alpha, \beta)=(0,1 / 2 T)$, of $\mathbf{x}(t)$. This is done through the exploitation of the temporal mean of the first correlation matrix of the extended derotated model $\widetilde{\mathbf{x}}_{d}(t) \triangleq\left[\mathbf{x}_{d}^{T}(t), \mathbf{x}_{d}^{H}(t)\right]^{T}$, or equivalently of $\mathbf{x}_{F_{2}}(t) \triangleq$ $\left[\mathbf{x}^{T}(t), e^{j 2 \pi t / 2 T} \mathbf{x}^{H}(t)\right]^{T}=j^{t / T} \widetilde{\mathbf{x}}_{d}(t)$, also called two-input FRESH model in [10]. However, for $\Delta_{f}=0$, the two most energetic conjugate SO cyclic frequencies of a $\mathrm{QR} C \mathrm{CI}$ are $\beta_{d}=0$ and $\beta_{d}=-1 / T$, if the CCI is derotated, and $\beta= \pm 1 / 2 T$, without any derotation [21], which proves the sub-optimality of model $\widetilde{\mathbf{x}}_{d}(t)$, or $\mathbf{x}_{F_{2}}(t)$, which only exploits one of these two cyclic frequencies.

To exploit the information contained in at least $\alpha_{d}=0$, $\beta_{d}=0$ and $\beta_{d}=-1 / T$, or equivalently in at least $\alpha=0$ and $\beta= \pm 1 / 2 T$, a three-inputs FRESH model, $\mathbf{x}_{d F_{3}}(t) \triangleq$ $\left[\widetilde{\mathbf{x}}_{d}^{T}(t), e^{-j 4 \pi t / 2 T} \mathbf{x}_{d}^{H}(t)\right]^{T}$, or equivalently $\mathbf{x}_{F_{3}}(t) \triangleq\left[\mathbf{x}^{T}(t)\right.$, $\left.e^{j 2 \pi t / 2 T} \mathbf{x}^{H}(t), e^{-j 2 \pi t / 2 T} \mathbf{x}^{H}(t)\right]^{T}=j^{t / T} \mathbf{x}_{d F_{3}}(t)$, has been introduced in [10] for QR signals. It has been shown in [10] that the temporal mean of the first correlation matrices of $\mathbf{x}_{d F_{3}}(t)$ and $\mathbf{x}_{F_{3}}(t)$ exploit the information contained in $\alpha=0$, $\alpha= \pm 1 / T$ and $\beta= \pm 1 / 2 T$.

However, for $\Delta_{f} \neq 0$, the two most energetic conjugate SO cyclic frequencies of a QR CCI become $\beta_{d}=2 \Delta_{f}$ and $\beta_{d}=2 \Delta_{f}-1 / T$ for a derotated CCI, and $\beta=2 \Delta_{f} \pm 1 / 2 T$ without any derotation. This gives $\beta_{d} \neq 0$ if $\Delta_{f} \neq 0$ and $\Delta_{f} \neq 1 / 2 T$ and $\beta_{d} \neq-1 / T$ if $\Delta_{f} \neq 0$ and $\Delta_{f} \neq-1 / 2 T$. In other words, for $\Delta_{f} \neq 0$ and $\Delta_{f} \neq \pm 1 / 2 T$, both the two and three-inputs FRESH models and associated receivers give poor performance and extended two and three inputs models are required.

\section{Extended two and three inputs FRESH model}

The extended two-inputs FRESH model must exploit the information contained in $\beta_{d}=2 \Delta_{f}$ (or $\beta=2 \Delta_{f}+1 / 2 T$ ) instead of $\beta_{d}=0$ (or $\beta=1 / 2 T$ ). Similarly, the extended threeinputs FRESH model has to exploit the information contained in $\beta_{d}=2 \Delta_{f}$ and $\beta_{d}=2 \Delta_{f}-1 / T$ (or $\beta=2 \Delta_{f} \pm 1 / 2 T$ ) instead of $\beta_{d}=0$ and $\beta_{d}=-1 / T$ (or $\beta= \pm 1 / 2 T$ ). We then propose to exploit and to analyze the performance, both analytically and by simulations, of the extended two and threeinputs FRESH models respectively defined by

$$
\begin{aligned}
\mathbf{x}_{E F_{2}}(t) & \triangleq\left[\mathbf{x}^{T}(t), e^{j 2 \pi\left(2 \Delta_{f}+\frac{1}{2 T}\right) t} \mathbf{x}^{H}(t)\right]^{T} \\
& =j^{\frac{t}{T}}\left[\mathbf{x}_{d}^{T}(t), e^{j 4 \pi \Delta_{f} t} \mathbf{x}_{d}^{H}(t)\right]^{T} \triangleq j^{\frac{t}{T}} \mathbf{x}_{E d F_{2}}(t) \\
& =\sum_{k} j^{k} b_{k} \mathbf{g}_{E F_{2}, k}(t-k T)+\mathbf{n}_{E F_{2}}(t)
\end{aligned}
$$




$$
\begin{aligned}
\mathbf{x}_{E F_{3}}(t) & \triangleq\left[\mathbf{x}^{T}(t), e^{j 2 \pi\left(2 \Delta_{f}+\frac{1}{2 T}\right) t} \mathbf{x}^{H}(t), e^{j 2 \pi\left(2 \Delta_{f}-\frac{1}{2 T}\right) t} \mathbf{x}^{H}(t)\right]^{T} \\
& =j^{\frac{t}{T}}\left[\mathbf{x}_{d}^{T}(t), e^{j 4 \pi \Delta_{f} t} \mathbf{x}_{d}^{H}(t), e^{j 4 \pi\left(\Delta_{f}-1 / 2 T\right) t} \mathbf{x}_{d}^{H}(t)\right]^{T} \\
& \triangleq j^{\frac{t}{T}} \mathbf{x}_{E d F_{3}}(t)=\sum_{k} j^{k} b_{k} \mathbf{g}_{E F_{3}, k}(t-k T)+\mathbf{n}_{E F_{3}}(t)(10)
\end{aligned}
$$

Here, $\mathbf{n}_{E F_{2}}(t) \triangleq\left[\mathbf{n}^{T}(t), e^{j 2 \pi\left(2 \Delta_{f}+1 / 2 T\right) t} \mathbf{n}^{H}(t)\right]^{T}, \mathbf{n}_{E F_{3}}(t) \triangleq$ $\left[\mathbf{n}^{T}(t), e^{j 2 \pi\left(2 \Delta_{f}+1 / 2 T\right) t} \mathbf{n}^{H}(t), e^{j 2 \pi\left(2 \Delta_{f}-1 / 2 T\right) t} \mathbf{n}^{H}(t)\right]^{T}$, $\mathbf{g}_{E F_{2}, k}(t) \triangleq\left[\mathbf{g}^{T}(t), e^{j 4 \pi \Delta_{f} k T} e^{j 2 \pi\left(2 \Delta_{f}+1 / 2 T\right) t} \mathbf{g}^{H}(t)\right]^{T}$ and $\mathbf{g}_{E F_{3}, k}(t) \triangleq\left[\mathbf{g}^{T}(t), e^{j 4 \pi \Delta_{f} k T} e^{j 2 \pi\left(2 \Delta_{f}+1 / 2 T\right) t} \mathbf{g}^{H}(t)\right.$, $\left.e^{j 4 \pi \Delta_{f} k T} e^{j 2 \pi\left(2 \Delta_{f}-1 / 2 T\right) t} \mathbf{g}^{H}(t)\right]^{T}$.

\section{Generic Pseudo-MLSE RECEIVER}

\section{A. Pseudo-MLSE approach}

To extend, in an efficient original way and for an arbitrary propagation channel, the two and three-inputs FRESH SAIC/MAIC receivers for QR signals with differential FO, and to analyze the impact of this FO on the performance, we use the continuous time pseudo-maximum likelihood sequence estimation (MLSE) approach, introduced recently in [10], and we apply it to the models (9) and (10) respectively. This approach consists in computing the continuous time MLSE receiver from (9) or (10), assuming that the associated two or three-inputs FRESH total noise, $\mathbf{n}_{E F_{2}}(t)$ or $\mathbf{n}_{E F_{3}}(t)$, is Gaussian, circular and stationary.

\section{B. Generic extended (E) pseudo-MLSE receiver}

We denote by $\mathbf{x}_{E F_{M}}(t)$ and $\mathbf{n}_{E F_{M}}(t)$ the generic extended $M(M=1,2,3)$ inputs FRESH observation and associated FRESH total noise vectors respectively. We assume that $\mathbf{x}_{E F_{1}}(t)$ and $\mathbf{n}_{E F_{1}}(t)$ correspond to $\mathbf{x}(t)$ and $\mathbf{n}(t)$ respectively. Assuming a stationary, circular and Gaussian generic extended FRESH total noise $\mathbf{n}_{E F_{M}}(t)$, it is shown in [22], [23] that the sequence $\mathbf{b} \triangleq\left(b_{1}, \ldots, b_{K}\right)$ which maximizes its likelihood from $\mathbf{x}_{E F_{M}}(t)$ is the one which minimizes the following criterion ${ }^{1}$

$$
\Lambda(\mathbf{b})=\sum_{k=1}^{K} \sum_{k^{\prime}=1}^{K} b_{k} b_{k^{\prime}} r_{k, k^{\prime}}-2 \sum_{k=1}^{K} b_{k} z_{E F_{M}}(k)
$$

where $z_{E F_{M}}(k) \triangleq \Re\left[j^{-k} y_{E F_{M}}(k)\right]$ with

$$
\begin{gathered}
y_{E F_{M}}(k)=\int \mathbf{g}_{E F_{M}, k}^{H}(f)\left[\mathbf{R}_{n, E F_{M}}^{0}(f)\right]^{-1} \mathbf{x}_{E F_{M}}(f) e^{j 2 \pi f k T} d f \quad(12) \\
r_{k, k^{\prime}}=j^{k^{\prime}-k} \int \mathbf{g}_{E F_{M}, k}^{H}(f)\left[\mathbf{R}_{n, E F_{M}}^{0}(f)\right]^{-1} \mathbf{g}_{E F_{M}, k^{\prime}}(f) e^{j 2 \pi f\left(k-k^{\prime}\right) T} d f .
\end{gathered}
$$

Here, $\mathbf{R}_{n, E F_{M}}^{0}(f)$ is the Fourier transform of (6), where $\alpha_{i}$ and $\mathbf{n}(t)$ are replaced by 0 and $\mathbf{n}_{E F_{M}}(t)$ respectively, whereas $\mathbf{g}_{E F_{M}, k}(f)$ corresponds to $\mathbf{g}(f)$, for $M=1$.

\footnotetext{
${ }^{1}$ All Fourier transforms of vectors $\mathbf{x}$ and matrices $\mathbf{X}$ use the same notation where $t$ or $\tau$ is simply replaced by $f$.
}

\section{Interpretation of the E-pseudo-MLSE receiver}

We deduce from (12) that $y_{E F_{M}}(k)$ is the sampled version, at time $t=k T$, of the output of the filter whose frequency response is

$$
\mathbf{w}_{E F_{M}, k}^{H}(f) \triangleq\left(\left[\mathbf{R}_{n, E F_{M}}^{0}(f)\right]^{-1} \mathbf{g}_{E F_{M}, k}(f)\right)^{H}
$$

and whose input is $\mathbf{x}_{E F_{M}}(t)$. The structure of the extended $M$ inputs pseudo-MLSE receiver is then depicted at Fig. 1. It is composed of the TI $M$ inputs filter (14), followed by a sampling at the symbol rate, a derotation operation, a real part capture and a decision box implementing the Viterbi algorithm.

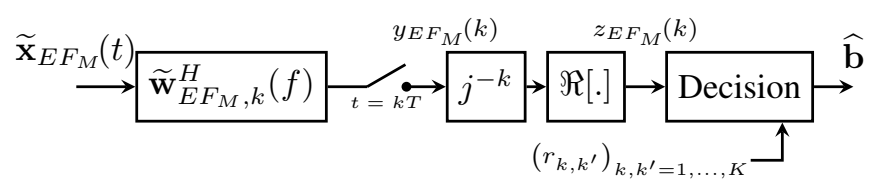

Fig. 1. Extended $M$ inputs pseudo-MLSE receiver

\section{SINR at the output of the E-pseudo-MLSE receiver}

For real-valued symbols $b_{k}$, the symbol error rate (SER) at the output of the extended $M$ inputs pseudo-MLSE receiver is directly linked to the signal to interference plus noise ratio (SINR) on the current symbol before decision, i.e. at the output $z_{E F_{M}}(n)$ [24, Sec 10.1.4], while the inter-symbol interference is processed by the decision box. For this reason, we compute this output SINR and we will analyse its variations in section IV. It is easy to verify from (1), (9), (10), (12) and (13) that $z_{E F_{M}}(n)$ can be written as

$$
z_{E F_{M}}(n)=b_{n} r_{n, n}+\sum_{k \neq n} b_{k} \Re\left[r_{n, k}\right]+z_{n, E F_{M}}(n)
$$

where $z_{n, E F_{M}}(n) \triangleq \Re\left[j^{-n} y_{n, E F_{M}}(n)\right]$ and $y_{n, E F_{M}}(n)$ is defined by (12) for $k=n$ with $\mathbf{n}_{E F_{M}}(f)$ instead of $\mathbf{x}_{E F_{M}}(f)$. The SINR on the current symbol is then defined by

$$
\operatorname{SINR}_{M, n} \triangleq \frac{\pi_{b} r_{n, n}^{2}}{\mathrm{E}\left[\Re\left[j^{-n} y_{n, E F_{M}}(n)\right]^{2}\right]} .
$$

where $\pi_{b} \triangleq \mathrm{E}\left[b_{n}^{2}\right]$. To analyze this $\operatorname{SINR}_{M, n}$ for particular scenarios, it is necessary to compute $r_{n, n}$ from (13) and $\mathrm{E}\left[\Re\left[j^{-n} y_{n, E F_{M}}(n)\right]^{2}\right]$ from the SO cyclic statistics of $y_{n, E F_{M}}(n)$. The latter statistics can be computed from (14) and the SO cyclic statistics of $\mathbf{n}_{E F_{M}}(t)$, themselves function of (6) and (7). The detailed way to derive the $\mathrm{SINR}_{M, n}$ from the cyclic statistics of $\mathbf{n}_{E F_{M}}(t)$ is tedious and not reported in this paper due to a lack of space. It will be presented elsewhere. For this reason, we just present and analyze in section IV, for particular scenarios, the final analytical expressions of $\mathrm{SINR}_{M, n}$.

\section{SINR ANALYSIS For ONE CCI}

\section{A. Assumptions}

To show the effectiveness of our pseudo-MLSE receiver, acting on (9) and (10) with respect to the conventional receiver and the better performance of (10) with respect to (9), we assume that the total noise is composed of one multi-user CCI 
and a background noise. We assume a raised cosine pulse shaping filter $v(t)$ with a roll-off $\gamma$. The SOI and CCI have the same bandwidth $B=(1+\gamma) / T$, and spectrally overlap if $0 \leq\left|\Delta_{f}\right| T \leq 1+\gamma$, as illustrated in Fig. 2, what we assume in the following. Due to space limitations, we limit the analysis to deterministic propagation channels with no delay spread such that

$$
\mathbf{h}(t)=\mu \delta(t) \mathbf{h} \quad \text { and } \quad \mathbf{h}_{I}(t)=\mu_{I} \delta\left(t-\tau_{I}\right) \mathbf{h}_{I} .
$$

Here, $\mu$ and $\mu_{I}$ control the amplitude of the SOI and CCI, $\delta(t)$ is the Dirac pulse, $\tau_{I}$ is the delay of the CCI with respect to the SOI whereas $\mathbf{h}$ and $\mathbf{h}_{I}$, such that $\mathbf{h}^{H} \mathbf{h}=\mathbf{h}_{I}^{H} \mathbf{h}_{I}=N$, are the channel vectors of the SOI and CCI.

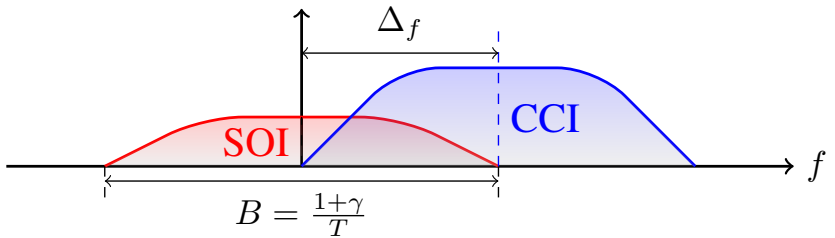

Fig. 2. Spectral representation of the SOI and CCI

\section{B. SINR computations and analysis}

Under the previous assumptions, analytical interpretable expressions of the SINRs (16) are only possible for a zero roll-off. In this case, we denote by $\pi_{s} \triangleq \mu^{2} \pi_{b}, \pi_{I} \triangleq \mu_{I}^{2} \pi_{e}$ and $\eta_{2}$ the power of the SOI, the CCI (for $\Delta_{f}=0$ ) and the background noise per antenna at the output of the pulse shaping matched filter respectively, $\pi_{e} \triangleq \mathrm{E}\left[e_{n}^{2}\right], \varepsilon_{s} \triangleq \pi_{s} \mathbf{h}^{H} \mathbf{h} / \eta_{2}$ and $\varepsilon_{I} \triangleq \pi_{I} \mathbf{h}_{I}^{H} \mathbf{h}_{I} / \eta_{2}$. Moreover, assuming $N=1$ and a strong CCI $\left(\varepsilon_{I} \gg 1\right)$ for models (9), (10) and also for model (1) if $\Delta_{f} \neq 0$, we obtain after tedious computations not reported here

$$
\begin{array}{rlr}
\mathrm{SINR}_{1} & \approx 2 \varepsilon_{s}\left|\Delta_{f}\right| T ; & \Delta_{f} \neq 0 \\
\mathrm{SINR}_{1} & =\frac{2 \varepsilon_{s}}{1+\varepsilon_{I}\left[1+\cos \left(2 \phi_{I s}\right) \cos \left(\frac{\pi \tau_{I}}{T}\right)\right]} ; \Delta_{f}=0 & \text { (19) } \\
\mathrm{SINR}_{2} & \approx 2 \varepsilon_{s}\left|\Delta_{f}\right| T ; & 0.5 \leq\left|\Delta_{f}\right| T \leq 1 \\
\mathrm{SINR}_{2} & \approx \frac{\varepsilon_{s}\left[1+2\left|\Delta_{f}\right| T\right]}{2} ; \quad 0.25 \leq\left|\Delta_{f}\right| T \leq 0.5 \\
\mathrm{SINR}_{2, n} & \approx 2 \varepsilon_{s}\left[1-0.5\left\{1+\left|\Delta_{f}\right| T+\left(1-4\left|\Delta_{f}\right| T\right) \cos \left(\Psi_{s I, n}\right)\right\}\right] \\
0 & \leq\left|\Delta_{f}\right| T \leq 0.25 ; \quad\left(\Delta_{f}, \Psi_{s I, n}\right) \neq(0, k \pi) \\
\mathrm{SINR}_{2} & \approx \frac{9 \varepsilon_{s}}{2 \varepsilon_{I}\left[3+2 \cos \left(4 \phi_{I s}\right)\right]} ;\left(\Delta_{f}, \Psi_{s I, n}\right)=(0, k \pi) & (23) \\
\mathrm{SINR}_{3, n} & \approx 2 \varepsilon_{s}\left[1-\frac{c_{1}+c_{2}\left|\Delta_{f}\right| T+c_{3}\left(\left|\Delta_{f}\right| T\right)^{2}}{1+c_{4}\left|\Delta_{f}\right| T}\right] .
\end{array}
$$

Here $\phi_{I s} \triangleq \operatorname{Arg}\left(\mathbf{h}_{I}^{H} \mathbf{h}\right)$ and $\Psi_{s I, n} \triangleq\left[-\phi_{I s}+2 \pi \Delta_{f}(n T-\right.$ $\left.\left.\tau_{I}\right)-\pi \tau_{I} / 2 T\right]$. The $c_{i}$ quantities, $1 \leq i \leq 4$, are complex coefficients, functions of $\phi_{I s}, \Delta_{f}, T, n$ and $\tau_{I}$, whose expressions are different for $\left|\Delta_{f}\right| T \in[0,0.25],[0.25,0.5],[0.5,0.75]$ and $[0.75,1]$ respectively. As these expressions are complicate, they are not given in the paper by lack of space.

A receiver performs $\mathrm{SAIC}$ as $\varepsilon_{I} \rightarrow \infty$ at time $n T$ if the associated $\operatorname{SINR}_{M, n}$ does not converge toward zero. We deduce from (18) that the conventional receiver performs SAIC as long as there is a spectral discrimination between the sources $\left(\Delta_{f} \neq 0\right)$. In this case, it is not sensitive to the phase of the signals and the output SINR does not depend on $n$. The SINR is maximum and equal to $2 \varepsilon_{s}$, the one obtained without CCI, if the sources do not overlap $\left(\left|\Delta_{f}\right| T=1\right)$. Otherwise, the output SINR strongly decreases as the overlap between the sources strongly increases. For a complete overlap $\left(\Delta_{f}=0\right)$, (19) shows that SAIC at the output of the conventional receiver is generally no longer possible, except when $\left(\tau_{I} / T, \phi_{I s}\right)=\left(2 k_{1},\left(2 k_{2}-1\right) \pi / 2\right)$ or $\left(2 k_{1}+1, k_{2} \pi\right)$, where $k_{1}$ and $k_{2}$ are integers.

Moreover, we deduce from (20) and (21) that for a spectral overlap which is less than $75 \%$ and for a strong CCI, the extended two-inputs WL FRESH receiver still performs SAIC thanks to a spectral discrimination between the sources only. Nevertheless, while its performance correspond to those of the conventional receiver for a spectral overlap which is less than $50 \%$, it has better performance than the conventional receiver for a spectral overlap comprised between $50 \%$ and $75 \%$. For a spectral overlap which is greater than $75 \%$ and for a strong CCI, (22) shows that the extended two-inputs WL FRESH receiver discriminates the sources spectrally and by phases and the output SINR depends on the differential phase of the sources and then on $n$. It completely cancels the CCI as long as there is at least one of the two discriminations (spectrum or phase) between the sources $\left(\Delta_{f}, \Psi_{s I, n}\right) \neq(0, k \pi)$. However (22) shows that the relative weight of the phase discrimination with respect to the spectral one increases with the spectral overlap. In other words, the phase discrimination takes over from the spectral one when the latter becomes too weak, which generates better performance than the conventional receiver for SAIC. Note that such an analysis from analytical SINR results has never been reported elsewhere.

The complicate expression (24) of $\mathrm{SINR}_{3, n}$ that we have obtained allows us to show that for a strong CCI, the extended three-inputs WL FRESH receiver performs SAIC thanks to a spectral discrimination only when the spectral overlap is less than $25 \%$, with $\mathrm{SINR}_{3} \geq \mathrm{SINR}_{2}$. For a spectral overlap greater than $25 \%$, the extended three-inputs WL FRESH receiver performs SAIC thanks to both a spectral and a phase discrimination between the sources with an increasing weight of the latter as the overlap increases. In all cases, we have shown that $\mathrm{SINR}_{3} \geq \mathrm{SINR}_{2}$, hence the great interest of the extended three inputs receivers with respect to the two-inputs one.

To give a statistical perspective of these results for arbitrary values of $\gamma$, we now assume that $\phi_{I s}, \pi \tau_{I} / 2 T$ and $n$ are independent random variables uniformly distributed on $[0,2 \pi],[0,2 \pi]$ and $\left[0,\left\lfloor 1 /\left|\Delta_{f}\right| T\right\rfloor\right]$ for $\Delta_{f} \neq 0$ respectively. Under these assumptions, choosing $\varepsilon_{s}=10 \mathrm{~dB}$ and $\varepsilon_{I}=20 \mathrm{~dB}$, Figures 3 and 4 show, for $\gamma=0$ and 0.5 respectively, $M=1,2,3$, and $\left|\Delta_{f}\right| T=0,0.25$ and 0.5 , $\operatorname{Pr}\left[\left(\mathrm{SINR}_{M, n} / 2 \varepsilon_{s}\right) \mathrm{dB} \geq x \mathrm{~dB}\right] \triangleq p_{M}(x)$ as a function of $x(\mathrm{~dB})$, where $\operatorname{Pr}[$.$] means probability. Note, whatever$ $\gamma$ and $\left|\Delta_{f}\right| T$, both good performance of the extended $M$ - 
inputs WL FRESH receivers for $M=2$ and 3 and increasing performance with $M$ of these receivers, proving the interest of (10) with respect to (9). Note also, for a given value of $M$, increasing performance with $\left|\Delta_{f}\right| T$ of the $M$-inputs WL FRESH receiver. For example, for $\gamma=0.5$ and $x=-3 \mathrm{~dB}$, we note that $p_{3}(x)=50 \%, 62 \%, 85 \%$ for $\left|\Delta_{f}\right| T=0,0.25$ and 0.5 respectively, showing very good performance of the extended 3-inputs WL FRESH receiver whatever $\left|\Delta_{f}\right| T$.

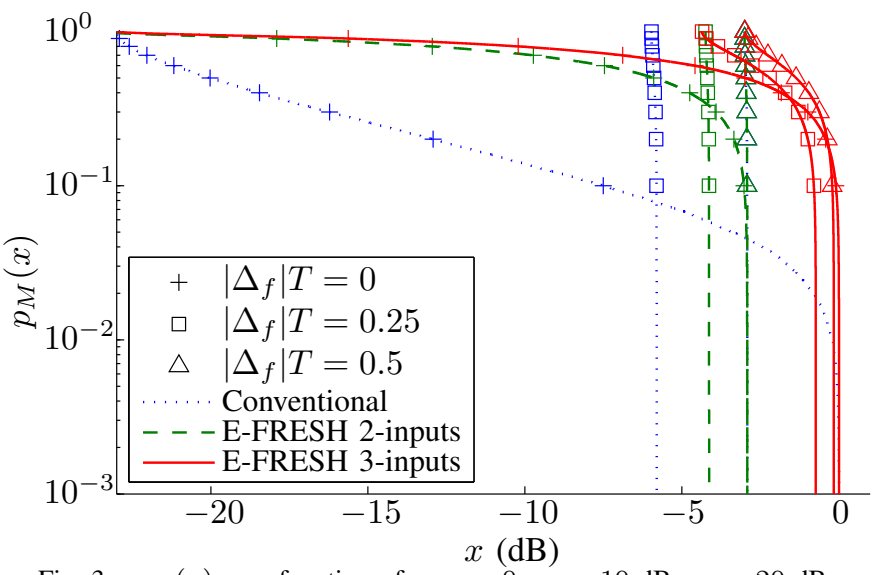

Fig. 3. $p_{M}(x)$ as a function of $x, \gamma=0, \varepsilon_{s}=10 \mathrm{~dB}, \varepsilon_{I}=20 \mathrm{~dB}$.

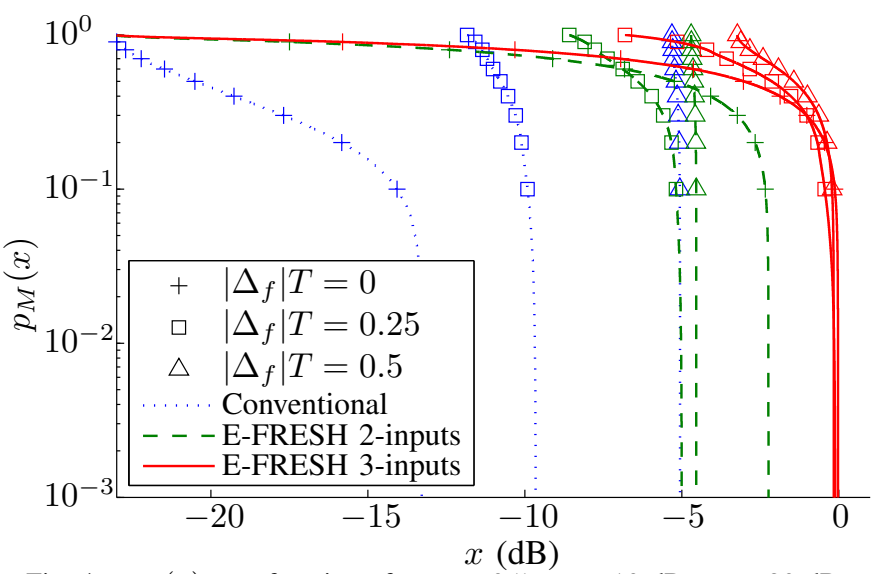

Fig. 4. $p_{M}(x)$ as a function of $x, \gamma=0.5, \varepsilon_{s}=10 \mathrm{~dB}, \varepsilon_{I}=20 \mathrm{~dB}$.

\section{CONCLUSION}

The two and three-inputs SAIC/MAIC WL FRESH receivers have been extended, for arbitrary propagation channels and from a MLSE-based approach, for QR signals having differential FO. Performance of the proposed receivers have been analyzed for deterministic channels with no delay spread, both analytically and by simulations, enlightening the impact of the FO parameter on the performance. Roles of spectral and phase discrimination between the sources have been explained for one receive antenna, from original analytical expressions of the output SINR. Finally, it has been shown that contrary to the conventional receiver, the proposed WL FRESH receivers have good performance whatever the value of the FO, increasing with the number $M$ of inputs. These receivers may open new perspectives, for ICI mitigation of FBMC-OQAM waveforms in particular.

\section{REFERENCES}

[1] W.M. Brown and R.B. Crane, "Conjugate linear filtering", IEEE Trans. Inf. Theory, vol. 15, no. 4, pp. 462-465, July. 1969.

[2] W.A. Gardner, "Cyclic Wiener filtering: theory and method", IEEE Trans. Commun., vol. 41, no. 1, pp. 151-163, Jan. 1993.

[3] B. Picinbono and P. Chevalier, "Widely linear estimation with complex data", IEEE Trans. Signal Process., vol. 43, no. 8, pp. 2030-2033, Aug. 1995.

[4] P. Chevalier, "Optimal array processing for non stationary signals", Proc ICASSP, pp. 2868-2871, Atlanta (USA), May 1996.

[5] B. Picinbono, "On Circularity", IEEE Trans. Signal Process., vol. 42, no. 12, pp. 3473-3482, Dec 1994.

[6] H. Trigui and D.T.M. Slock, "Performance bounds for cochannel interference cancellation within the current GSM standard", Signal Processing, Elsevier, vol. 80, pp. 1335-1346, 2000.

[7] P. Chevalier and F. Pipon, "New Insights into optimal widely linear array receivers for the demodulation of BPSK, MSK and GMSK signals corrupted by noncircular interferences - Application to SAIC", IEEE Trans. Signal Process., vol. 54, no. 3, pp. 870-883, March 2006.

[8] R. Meyer, W.H. Gerstacker, R. Schober, and J.B. Huber, "A single antenna interference cancellation algorithm for increased GSM capacity", IEEE Trans. Wireless Commun., vol. 5, no. 7, pp. 1616-1621, July 2006.

[9] R. Chauvat, P. Chevalier and J.P. Delmas, "How to make quasi-rectilinear signals (MSK, GMSK, OQAM) almost equivalent to rectilinear ones (BPSK, ASK) for WL filtering in the presence of CCI", ITG Workshop on Smart Antennas (WSA'15), Ilmenau (Germany), March 2015.

[10] P. Chevalier, R. Chauvat, and J.P. Delmas, "Quasi-rectilinear (MSK, GMSK, OQAM) co-channel interference mitigation by three inputs WL FRESH filtering", Proc. ICASSP, Brisbane (Australia), Apr. 2015.

[11] J.P. Delmas, S. Sallem and P. Chevalier, "Sensitivity of SAIC and MAIC Concepts to Residual Frequency Offsets", Proc. European Signal Processing Conference (EUSIPCO'10), Alborg (Denmark), Aug. 2010.

[12] G. Wunder, P. Jung, M. Kasparick, T. Wild, F. Schaich, Y. Chen, S. Ten Brink, I. Gaspar, N. Michailow, A. Festag, L. Mendes, N. Cassiau, D. Ktenas, M. Dryjanski, S. Pietrzyk, B. Eged, P. Vago and F. Wiedmann, "5GNOW: Non-orthogonal asynchronous waveforms for future mobile applications", IEEE Com. Mag., pp. 97-105, Feb. 2014.

[13] M. Caus and A.I. Perez-Neira, "Multi-stream transmission for highly frequency-selective channels in MIMO-FBMC/OQAM systems", IEEE Trans. Signal Process., vol. 62, no. 4, pp. 786-796, Feb. 2014.

[14] Y. Cheng and M. Haardt, "Widely linear processing in MIMO FBMC/OQAM systems", Intern. Symposium on Wireless Communication Systems (ISWCS'13), pp. 743-747, Aug. 2013.

[15] S. Josilo, M. Narandzic, S. Tomic, and S. Nedic, "Widely linear filtering based kindred CCI suppression in FBMC waveforms", Intern. Symposium on Wireless Communication Systems (ISWCS'14), Barcelona, Aug. 2014

[16] W.A. Gardner and C.W. Reed, "Making the most out of spectral redundancy in GSM: Cheap CCI suppression", Proc. ASILOMAR Conf., pp. 883-889, Pacific-Grove, 2001.

[17] A. Mirbagheri, K.N. Plataniotis, and S. Pasupathy, "An enhanced Widely Linear CDMA receiver with OQPSK modulation", IEEE Trans. Commun., vol. 54, no. 2, pp. 261-272, Feb. 2006.

[18] A.U.H. Sheikh and F. Hendessi, "FRESH-DFE: a new structure for interference cancellation", Wireless Personal Communications Journal, vol. 44, pp. 101-118, 2008.

[19] R. Chauvat, P. Chevalier, J.P. Delmas, "Widely linear FRESH receiver for SAIC/MAIC with frequency offsets", Intern. Symposium on Wireless Communications Systems (ISWCS'15), Brussels (Belgium), Aug. 2015.

[20] W.H. Gerstacker, R. Schober, and A. Lampe, "Receivers with widely linear processing for frequency-selective channels", IEEE Trans. Commun., vol. 51, no. 9, pp. 1512-1523, Sept. 2003.

[21] D. Vucic and M. Obradovic, "Spectral Correlation evaluation of MSK and Offset QPSK modulation", Signal Proc., vol. 78, pp. 363-367, 1999.

[22] S. Sallem, J.P. Delmas, and P. Chevalier, "Optimal SIMO MLSE receivers for the detection of linear modulation corrupted by noncircular interference," IEEE workshop on Statistical Signal Processing (SSP'2012), Ann Arbor (USA), Aug. 2012.

[23] G. Ungerboeck, "Adaptive maximum likelihood receiver for carriermodulated data transmission systems", IEEE Trans. Commun., vol. 22, no. 5, pp. 624-636, May 1974.

[24] J.G. Proakis, Digital Communications, McGraw Hill Series in Electrical and Computer Engineering, 4th Edition, 2001. 